SLAC-PUB-11235

May 2005

\title{
EMITTANCE DILUTION DUE TO MANY-BAND LONG-RANGE DIPOLE WAKEFIELDS IN THE INTERNATIONAL LINEAR COLLIDER MAIN LINACS ${ }^{\dagger}$
}

\author{
R.M. Jones ${ }^{\dagger}$ and N. Baboi ${ }^{\ddagger}$ \\ ${ }^{\dagger}$ Stanford Linear Accelerator Center, \\ 2575 Sand Hill Road, Menlo Park, CA, 94025 \\ ${ }^{\ddagger}$ DESY, Notkestraße 85 D-22603 Hamburg, Germany
}

\begin{abstract}
We investigate the emittance dilution that occurs due to long range wakefields in the ILC (International Linear Collider) L-band linacs. In previous simulations we have focused upon the largest kick factors (proportional to the transverse fields which transversely kick the beam off axis) for the first three pass-bands. Here we supplement these calculations with an additional four bands. We include seven pass-bands in our simulations with the upper dipole frequencies extending a little higher than $4 \mathrm{GHz}$. Higher order dipole modes in the first three pass-bands are damped by carefully orientating higher order mode couplers at both ends of each cavity. Here we investigate the impact of upper band modes on the beam dynamics.
\end{abstract}

Paper presented at the $21^{\text {st }}$ Particle Accelerator Conference (PAC 2005),

Knoxville, TN, $16^{\text {th }}-20^{\text {th }}$ May 2005 


\title{
EMITTANCE DILUTION DUE TO MANY-BAND LONG-RANGE DIPOLE WAKEFIELDS IN THE INTERNATIONAL LINEAR COLLIDER MAIN LINACS
}

\author{
R.M. Jones ${ }^{\dagger}$; SLAC, Stanford, CA 94309, USA and N. Baboi; DESY, Hamburg, Germany
}

\begin{abstract}
We investigate the emittance dilution that occurs due to long range wakefields in the ILC (International Linear Collider) [1] L-band linacs. In previous simulations [2] we have focused upon the largest kick factors (proportional to the transverse fields which transversely kick the beam off axis) for the first three pass-bands. Here we supplement these calculations with an additional four bands. We include seven pass-bands in our simulations with the upper dipole frequencies extending a little higher than $4 \mathrm{GHz}$. Higher order dipole modes in the first three pass-bands are damped by carefully orientating higher order mode couplers at both ends of each cavity. Here we investigate the impact of upper band modes on the beam dynamics.
\end{abstract}

\section{INTRODUCTION}

In the operation of any linear collider it is important to maintain the emittance of the beam as it travels down the main linacs in order to maximize the luminosity of the colliding beams at the interaction point. In the ILC we consider colliding 2820 bunches and accelerating them down the main linacs with an initial design gradient of 23.4 MV/m (based on the TESLA design [3]). Although there are alternative options which allow for an increased accelerating gradient. Some typical parameters of the ILC main linacs are given in table 1 . As the main linacs are superconducting then little power is lost in heating the walls of the cavity and we can afford to have a long pulse train of $950 \mu$ s consisting of 2820 bunches each separated from their neighbors by 336.9 ns.

\begin{tabular}{|l|l|l|}
\hline \multicolumn{1}{|c|}{ Quantity } & Symbol & \multicolumn{1}{c|}{ L } \\
\hline Accelerating freq. $(\mathrm{GHz})$. & $\mathrm{f}_{\mathrm{acc}}$ & 1.300 \\
\hline Loaded gradient $(\mathrm{MV} / \mathrm{m})$ & $\mathrm{G}_{\mathrm{acc}}$ & 28 \\
\hline Bunch train length $\left(\mathrm{T}_{\mathrm{fill}}\right)$ & $\mathrm{T}_{\mathrm{b}}$ & 2.88 \\
\hline Bunch spacing $\left(\mathrm{T}_{\mathrm{RF}}\right)$ & $\mathrm{T}_{\mathrm{bb}}$ & 438 \\
\hline Charge per bunch $\left(10^{10}\right)$ & $\mathrm{N}_{\mathrm{e}}$ & 2 \\
\hline Structure Iris radius $\left(\lambda_{\mathrm{RF}}\right)$ & $\mathrm{A}$ & 0.152 \\
\hline Bunch length $(\mu \mathrm{m})$ & $\mathrm{\sigma}_{\mathrm{z}}$ & 300 \\
\hline Pulse rate & $\mathrm{f}_{\text {rep }}$ & 5 \\
\hline
\end{tabular}

Table 1: Fundamental L-band ILC RF parameters

The accelerated bunches of charged particles experience a wakefield that has both long range and short range components. The former component arises from the electromagnetic field of the bunch itself and the latter component is due to the electromagnetic field experienced by bunches trailing the accelerated bunches which drive the wake. These wakefields can dilute the emittance of the beams. At the very least, this can reduce the

† Supported by the U.S. DOE grant number DE-AC02-76SF00515 luminosity, which for flat beams $\left(\varepsilon_{\mathrm{x}, \mathrm{n}} / \varepsilon_{\mathrm{y}, \mathrm{n}}=400\right)$ is proportional to $\mathrm{P}_{\mathrm{b}} / \sqrt{\varepsilon_{\mathrm{y}, \mathrm{n}}}$, where $\mathrm{P}_{\mathrm{b}} \propto \mathrm{f}_{\text {rep }} \mathrm{n}_{\mathrm{b}} \mathrm{q}$ is the beam power and $\varepsilon_{\mathrm{y}, \mathrm{n}}\left(\varepsilon_{\mathrm{x}, \mathrm{n}}\right)$ the normalized vertical (horizontal) emittance at the interaction point. For the ILC a high $\mathrm{P}_{\mathrm{b}}$ is achieved by compensating the low pulse repetition frequency, $\mathrm{f}_{\text {rep }}$, with a high bunch charge $\mathrm{q}$, and a large number of bunches $n_{b}$. In the worst case scenario the wakefield can give rise to a BBU (Beam Break Up) instability [4]. Thus, it is clear that the wakefields in the linacs must be damped to manageable values and this is achieved by providing each cavity with HOM (Higher Order Mode) couplers at each end and this is illustrated in fig. 1 . There will be close to 21,000 of these cavities in the collider. Each cavity consists of nine cells, operating

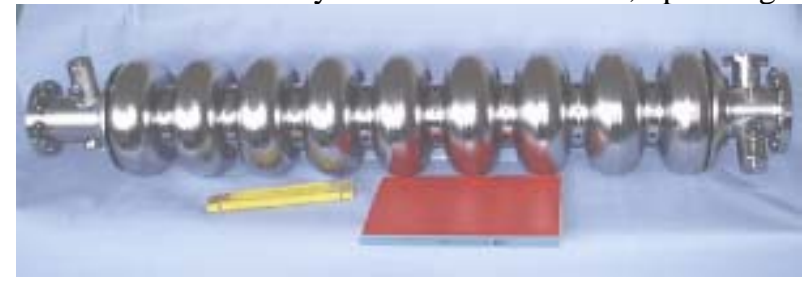

Figure 1: Fabricated nine-cell $\mathrm{Nb}$ TESLA cavity suitable for use in the ILC

in the standing wave mode and with a $\pi$ phase advance per cell.

The paper is organized such that the next section discusses the long-range wakes for 7 pass-bands and the penultimate main section entails particle tracking simulations to calculate the emittance dilution.

\section{TRANSVERSE WAKEFIELDS}

The long-range transverse wakefield [5], at a distance $s$ behind the first bunch, is calculated from the real part of the modal sum:

$$
\mathrm{W}(\mathrm{s})=2 \operatorname{Re}\left\{\sum_{\mathrm{n}=1}^{\mathrm{N}} \mathrm{K}_{\mathrm{n}} \mathrm{e}^{\mathrm{i} \omega_{\mathrm{n}} \mathrm{s} / \mathrm{c}} \mathrm{e}^{-\omega_{\mathrm{n}} \mathrm{s} / 2 \mathrm{Q}_{\mathrm{n}} \mathrm{c}}\right\} \mathrm{U}(\mathrm{s})
$$

where $\mathrm{N}$ is the number of modes, $\mathrm{U}(\mathrm{s})$ is the unit step function, the $\mathrm{n}^{\text {th }}$ mode has a quality factor of $\mathrm{Q}_{\mathrm{n}}$, a kick factor $K_{n}$ and a synchronous frequency $\omega_{n} / 2 \pi$. The kick factors and mode frequencies for the first seven bands are illustrated in fig 2. These kick factors have been computed with the code MAFIA [6] and are taken from [7]. The corresponding Q values are displayed in fig 3. These Qs consist of experimentally determined damped values from a 'cold' measurement [8] and are supplemented by MAFIA calculations [6] for the upper 4 bands. The Qs are normalized with respect to the fundamental mode $\mathrm{Q}\left(\sim 10^{10}\right)$. The most damaging kick factor is located at $2.575 \mathrm{GHz}$ and this is carefully 


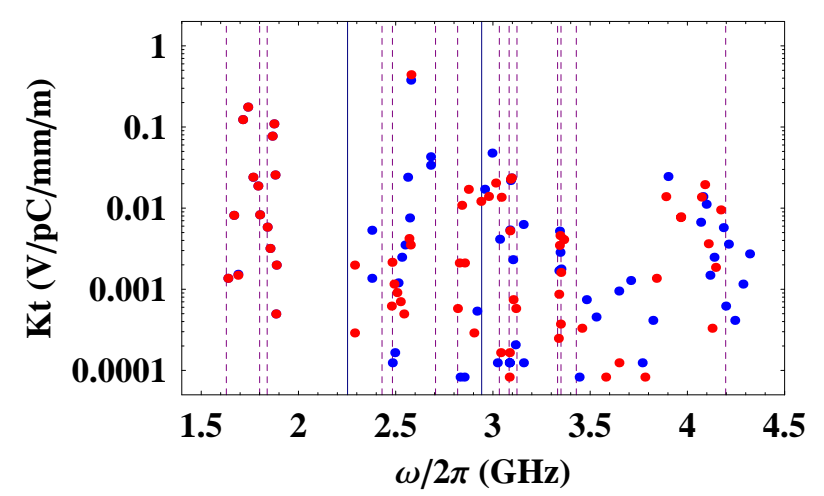

Figure 2: Kick factors for the first 6 dipole bands. (indicated by the dashed blue lines) The solid blue lines denote the $\mathrm{TE}_{11}$ and $\mathrm{TM}_{01}$ cut-off frequencies of the attached beam tubes. The blue dots refer to the TM-like modes and the red dots to the TE-like modes

damped down with HOM couplers to a $\mathrm{Q}$ of $5 \times 10^{4}$. Small errors in the dimensions of the cavities introduced during the process of fabricating several thousand cavities leads to slightly different dipole frequencies from cavityto-cavity. This effectively interleaves the frequencies of successive cavities and reduces the coherent transverse kick that the beam receives.

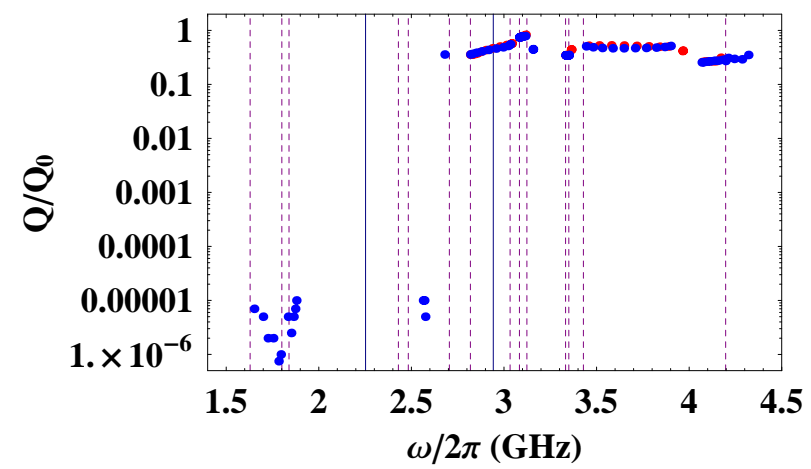

Figure 3: Modal Q values normalized with respect to the fundamental mode's Q. The blue dots refer to the TM-like modes and the red dots to the TE-like modes.

We subject the beam to these wakefields for several systematic frequency errors and $\mathrm{Q}$ values. We focus on the interaction of the beam with TM-like modes in the next section.

\section{BEAM DYNAMICS}

Past experience [9] has taught us that a good indication as to the maximum impact of the wakefield on disrupting the bunches of charged particles is given by the sum wakefield. The sum wakefield [9], defined at each bunch in question, as the sum of the wakes of all wakes at all previous bunches, is shown in fig 4 for the three different $\mathrm{Q}$ distributions. The abscissa refers to a percentage change in the bunch spacing and in practice it corresponds to a systematic shift in the frequencies of all cells in any given cavity. For each Q configuration we take a peak value close to the nominal bunch spacing (indicated by1,2

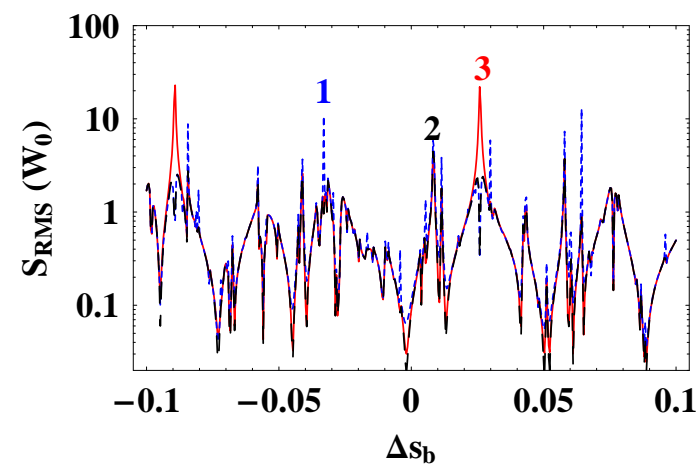

Figure 4: RMS of sum wakefield ( $\left.\mathrm{S}_{\mathrm{RMS}}\right)$ in units of $\mathrm{W}_{0}$ (= $0.13 \mathrm{~V} / \mathrm{pC} / \mathrm{mm} / \mathrm{m}$ ) versus percentage deviation in the bunch spacing from the design value for 3 different situations. The blue dashed curve is computed using the Qs given in fig 3. The black dashed curve corresponds to setting the Qs of the upper 4 band to $10^{6}$ and the red curve corresponds to the Qs of the red curve with the mode at $2.575 \mathrm{GHz}$ being assigned a value of $5 \times 10^{5}$.

and 3 in fig 4), and track the beam down the linac under these worst case scenarios. The results of these simulations, performed with the code LIAR [9], are presented in figs 5 and 6. Even for no HOM damping of the upper four bands (fig $4 \mathrm{a}$ and fig 5a) only $20 \%$ or so emittance dilution is expected to result. Furthermore, for the case of a badly damped mode with the largest kick factor of all bands (illustrated in fig 5c and 6c), no more than $15 \%$ dilution in the emittance occurs.

\section{CONCLUSIONS}

The upper band long-range dipole wakefields in the TESLA-style L-band accelerators are unlikely to give rise to significant emittance dilution for transverse injection offsets. The latest LL (Low loss) and re-entrant L-band cavities [11] have a modified geometry in order to sustain higher accelerating field gradient. The impact of the dipole modes on the beam dynamics requires investigation in these modified cavities. Furthermore, coupling of horizontal motion to vertical motion in several accelerator geometries can lead to serious emittance dilution. This effect is covered elsewhere for the TESLA-style geometry [12].

\section{REFERENCES}

[1] http://www.interactions.org/linearcollider/.

[2] R.M. Jones et al., EPAC04, SLAC-PUB-10556.

[3] R. Brinkmann et al. (editors), TESLA Report 2001-23.

[4] K. Yokoya, DESY Report 86-084, 1986.

[5] P.B. Wilson, SLAC-PUB-4547, 1989.

[6] The MAFIA Collaboration, CST, Darmstadt, 1994.

[7] R. Wanzenberg, TESLA 2001-33, Sept. 2001.

[8] G. Krepps, private communication.

[9] R.M. Jones et al., SLAC-PUB-8610, 2000.

[10] R. Assman et al., LIAR,SLAC-PUB AP-103, 1997.

[11] H. Padamsee, WOAA005, these proceedings.

[12] R.M. Jones et al., RPPP042, these proceedings. 

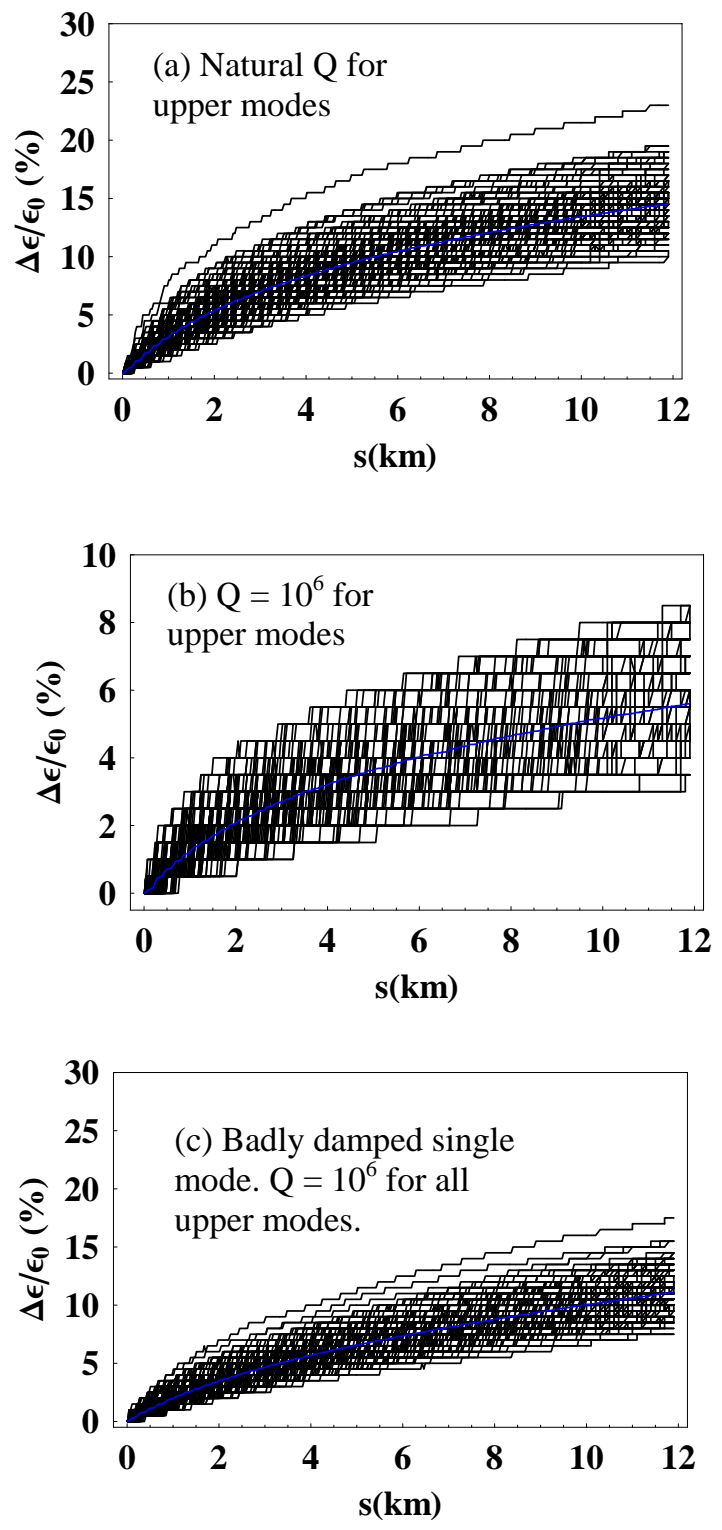

Figure 5: Emittance dilution down main L-band linacs for a one $\sigma$ injection offset of the full bunch train. In all these simulations we consider 500 bunches out of the full 2820 bunches in the train. Each of the individual black lines represents a particular machine of which there are 200. The average emittance dilution over 200 machines is indicated by the blue curve in all cases. Uppermost (a) is shown the emittance dilution that occurs when the upper band modes (all modes above $2.6 \mathrm{GHz}$ ) are not damped by the HOM couplers. The lower band modes are damped with Qs according to those given in fig.2. The middle (b) set of curves corresponds to well-damped lower band modes and upper-band modes are damped with a uniform $\mathrm{Q}$ of $10^{6}$ for each mode. The lowermost (c) set of curves corresponds to the damping in (b) with the $\mathrm{Q}$ of the mode at $2.575 \mathrm{GHz}$ being increased by a factor of 10 to $5 \times 10^{5}$.
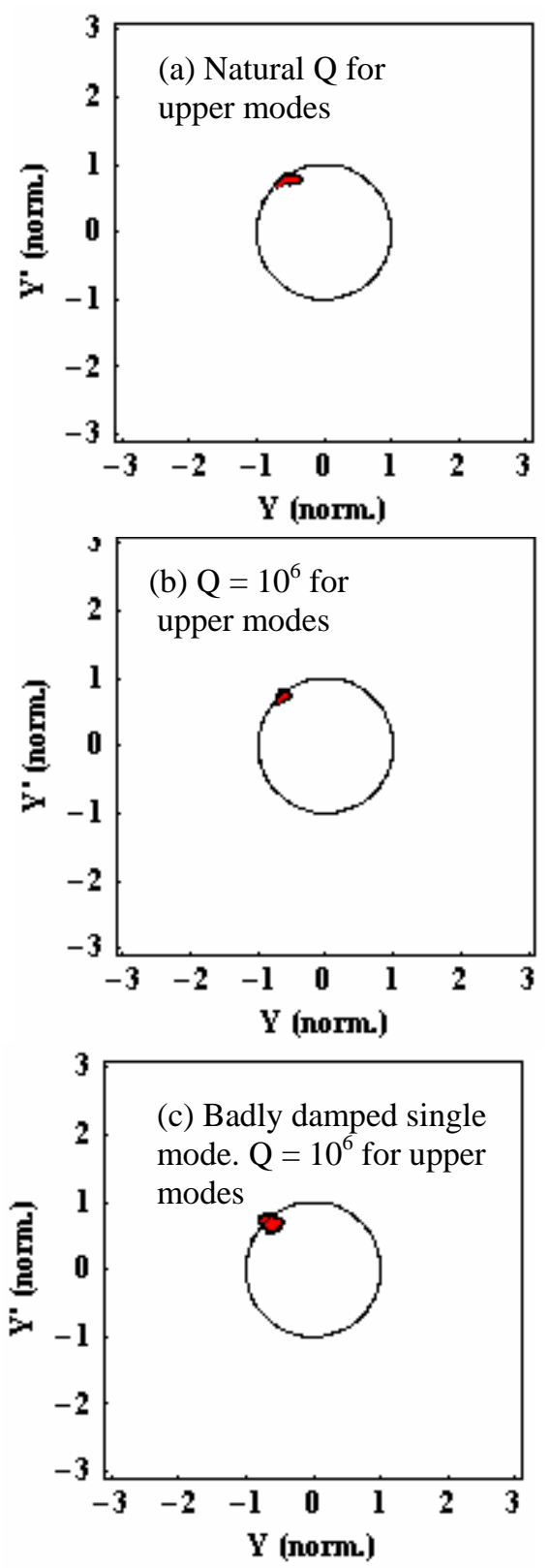

Figure 6: Vertical phase space for 500 bunches at the end of the main linacs for 200 machines (denoted by the black points). The mean of all 200 machines is denoted by the red dots. The damping conditions correspond to those presented adjacent in fig. 3 . The phase space of all bunches at the end of the linac is normalized with respect to the initial values of $\sigma=\left(\beta \varepsilon_{\mathrm{n}} \Omega \Upsilon\right)^{1 / 2}$ and $\sigma^{\prime}=\left[\left(1+\alpha^{2}\right) \varepsilon_{n} / \beta \Upsilon\right]^{1 / 2}$. Here, $\alpha, \beta$ and $\Upsilon$ are the characteristic Twiss parameters of the beam evaluated at the beginning of the linac, where the bunches are injected with $\varepsilon_{\mathrm{n}}$ ' the vertical emittance of 20 nm.rads. and the horizontal emittance is $8 \mu \mathrm{m}$.rads. The initial $\sigma \sim 10.1 \mu \mathrm{m}$ and $\sigma^{\prime} \sim 0.26 \mu$ rads and the corresponding values at the end of the linac are $\sigma \sim 1.5 \mu \mathrm{m}$ and $\sigma^{\prime} \sim 0.27 \mu$ rads 